\title{
Ribosomes, Bound and Unbound
}

ONE way to explain the finding that some ribosomes are bound to the membranes of the endoplasmic reticulum of eukaryotic cells whereas others seem to be free in the cytoplasm is to suppose that the bound ribosomes are concerned with the synthesis of proteins which are destined to be secreted from the cell. Writing in the current issue of Nature New Biology (232, 8; 1971), Baglioni and his colleagues at the Massachusetts Institute of Technology report that membrane-bound polyribosomes are assembled by a process in which the large $60 \mathrm{~S}$ ribosomal subunit first binds to the membrane, after which the small 40S subunit together with messenger RNA joins the complex. On page 102 of this issue of Nature, Williams and Rabin from University College, London, report their studies on the detachment from membranes of bound ribosomes by pharmacological reagents.

Their assay procedure is a devious method which depends on an enzymatic activity in endoplasmic reticulum that catalyses the rearrangement of disulphide bonds in proteins; the biological function of this enzyme is uncertain, but it may be concerned in ensuring the correct disulphide pairing and conformation of secreted and lysosomal proteins. When the correct pairing of the four disulphide bonds of the enzyme pancreatic ribonuclease is destroyed by reduction, the nuclease enzyme becomes unfolded and can be oxidized in such a way that the free - SH groups pair randomly. This version of the protein is not active, but the rearranging enzyme of endoplasmic reticulum can catalyse an interchange of the disulphide bonds of the nuclease to achieve thermodynamic equilibrium; this yields an active ribonuclease. Using this complex, and somewhat indirect, assay system, Williams and Rabin have previously found that the RNA content of microsomal preparations is inversely related to the rearrangement activity, and that addition of ribosomes in the presence of steroids inhibits all the apparent rearranging activity. Rabin and his colleagues have interpreted their observations to mean that when ribosomes bind to membranes they mask the activity of the "rearrangase" because the enzyme is located at the ribosome binding site. They have therefore used the activity of this enzyme-as measured by its effect on ribonuclease-to assay the extent of binding between ribosomes and membrane; it is this assay procedure on which they have based their results about how ribosomes bind to membranes and how steroid hormones and aflatoxin B influence this binding (see Nature New Biology, 230, 133 and 137 ; 1971).

It is reasonable to find that an enzyme, probably involved in the last stages of the biosynthesis of proteins for export, should be bound in some way to the ribosomes that make the proteins for export, and perhaps these ribosomes also make microsomal membrane proteins. Although, in the artificial assay system, the rearranging enzyme does not seem to work when the membranes are saturated with ribosomes, it is likely that in the living cell this enzyme is most active when the microsomal membrane is saturated with ribosomes. The significance of this paradox in the method is not clear.

A polycyclic carcinogenic poison, $0.12 \mathrm{mM}$ aflatoxin $B_{1}$, decreases the quantity of bound RNA and increases the apparent "rearrangase" activity. Membranes treated with
EDTA to remove ribosomes can reattach them subsequently, but membranes treated with aflatoxin cannot bind ribosomes again presumably because they have been damaged irreversibly. Corticosterone $(50 \mu \mathrm{g} / \mathrm{ml}$.) but not hydrocortisone slows down the rate of ribosome loss from membranes caused by aflatoxin. Corticosterone, however, enhances binding and hydrocortisone inhibits binding of ribosomes to membranes treated with EDTA.

Oestradiol, pregnanediol and corticosterone enhance the binding of male ribosomes to male membranes, and testosterone enhances the binding of female ribosomes to female membranes. But male hormones in male systems and female hormones in female systems are without effect in this test. It is not apparent whether this effect results from the endogenous oestrogens in females saturating "oestrogen" sites, thereby only allowing the opposite sex hormone to have an effect in this system in vitro.

In the work described on page 102 some carcinogens are shown to unbind ribosomes from membranes in contrast to some non-carcinogenic analogues which do not show this property. Oestradiol protects against unbinding by the active compounds. Unlike EDTA unbinding, the carcinogens have an irreversible effect and prevent the binding of ribosomes to smooth membranes in the presence of oestradiol.

There are no experimental reasons to equate the effects observed in this complex system with the physiological effects of these hormones. The observations reveal some interesting aspects of steroid hormone behaviour; but perhaps not the only ones. One school of thought holds that the primary physiological effect of steroid hormones may be to regulate the synthesis of RNA in the cell nucleus. Williams and Rabin may perhaps be observing an unusual tissue specific effect. If uterine microsomal preparations do not respond to oestrogens it would be difficult to argue that this is nonetheless a prime mode of oestrogen action. It will be very interesting to see the behaviour in castrated male rat liver.

An alternative possibility is that the prime effect of these sex hormones is indeed in the cell nucleus; but, in addition, these compounds which have an anabolic character enhance the binding of ribosomes to microsomal membranes, with the result that the synthesis of microsomal membrane protein is increased and consequently the capacity of the cell to synthesize molecules and to grow is greater.

The relevance of the observations to carcinogenesis is both more intriguing and less certain. A correlation has been established between a few carcinogens and unbinding of ribosomes from membranes, but acute and chronic poisons remove ribosomes from microsomal membranes. The experiments reported are all short term experiments. A striking feature of carcinogenesis is that it commonly requires lengthy administration before an effect is observed. The partial unbinding of ribosomes by the carcinogens is irreversible. It will be informative to hear what effect a prolonged change in the ratio of bound to unbound ribosomes has on the reproductive behaviour of liver cells. If bound ribosomes participate in the synthesis of the glycoproteins of the cell surface, then irreversible interference with the proper functioning of bound ribosomes may result in altered cell surface properties. 